\title{
Los efectos de la campaña para las elecciones generales españolas de $2011^{*}$
}

\author{
Enrique García Viñuela \\ Universidad Complutense de Madrid
}

\begin{abstract}
Resumen
Este artículo estima los efectos de la campaña para las elecciones generales españolas de 2011 sobre los resultados de los tres partidos más votados: el Partido Popular (PP), el Partido Socialista (PSOE) e Izquierda Unida (IU). Utilizando datos de un panel electoral del CIS, encuentro que el efecto dominante de la campaña fue el refuerzo de la intención de voto, en particular al PP, el partido de centro-derecha ganador de las elecciones. La edad y el nivel de información fueron las características personales de los votantes que propiciaron el refuerzo. Por el contrario, los cambios en la ideología y en la valoración de los líderes políticos promovieron los efectos de activación y conversión de la campaña, que fueron más intensos para los dos partidos de izquierda, el PSOE e IU. El efecto de conversión favoreció a IU, mientras que la activación del voto ideológico de sus partidarios permitió al PSOE contener la notable pérdida de apoyo en las urnas que tuvo en estas elecciones.
\end{abstract}

Palabras clave: elecciones, efectos de las campañas electorales, España.

Código JEL: D72.

\begin{abstract}
This paper estimates the effects of the campaign for the 2011 Spanish general election on the results of the three most voted parties: the right of center Popular Party (PP), the Socialist Party (PSOE) and the United Left (IU). Using data from a CIS election panel, I find that the reinforcement of the voting intention reported in the preelection survey was the strongest campaign effect, especially for the $P P$, the main opposition party and the winner of the election. Voters' age and information level were the personal traits that encouraged reinforcement. On the contrary, changes in ideology and the assessment of party leaders fostered the activation and conversion effects, which were more intense for the two parties on the left, the PSOE and the IU. The conversion effect benefited the IU, while the activation of the ideological vote of its partisans allowed the PSOE to limit the large electoral loss that underwent in the 2011 election.
\end{abstract}

Keywords: elections, campaign effects, Spain.

JEL classification: D72.

\section{Introducción}

Las acciones que emprenden los partidos para influir sobre los votantes antes de unas elecciones han atraído el interés de numerosos investigadores de la ciencia política y la elección pública. Los efectos de esas acciones sobre el comportamiento de los votantes es, sin embargo, un asunto controvertido en la literatura. Los primeros

\footnotetext{
* Agradezco a Joaquín Artés sus comentarios a una versión previa de este artículo.
} 
estudios empíricos sobre la influencia de las campañas electorales a mediados del siglo pasado (Lazarsfeld et al., 1944; Campbell et al., 1960), concluyeron que las campañas afectaban poco a los resultados electorales de los partidos.

La investigación más reciente se inclina por una influencia de las campañas bastante mayor. Por ejemplo, Rosentone y Hansen (1993), Holbrook (1996), y Franz y Rideout (2007) muestran que las actividades de los partidos y los candidatos durante la campaña aumentan la participación en las urnas. Los trabajos de Popkin (1991), Simon (2002) y Clinton y Lapinski (2004) detectan que los debates sobre los temas de la elección y las políticas que se proponen para resolverlos, influyen sobre el voto. Y Shaw (1999); Holbrook y McClurg (2005); Hillygus (2005) y McClurg y Holbrook (2009), encuentran que puede establecerse una relación entre la intensidad de la movilización política y los resultados electorales.

Usando datos de un panel con información sobre una misma muestra de individuos antes y después de la campaña electoral, Lazarsfeld et al.(1944) definieron los tres efectos de las campañas como refuerzo (de las preferencias políticas anteriores a la campaña), activación (del voto de los electores indecisos) y conversión (cambio en las preferencias de partido entre las dos encuestas del panel), comparando las preferencias de voto de los encuestados antes de la campaña con el voto declarado en la encuesta postelectoral. A estos tres efectos, Martínez i Coma (2008) añade la desactivación (de los electores que pensaban votar antes de la campaña, pero terminan por abstenerse). En este trabajo estimo estos cuatro efectos utilizando un panel de dos encuestas del Centro de Investigaciones Sociológicas (CIS) sobre las elecciones generales españolas de $2011^{1}$.

Las campañas no afectan por igual a todos los individuos. Éstos tienen características diferentes y no reaccionan de la misma manera al esfuerzo electoral de los partidos. Distintos autores (Kenny y McBurnett, 1994; Shaw, 1999; Criado, 2008), encuentran que la publicidad electoral es más eficaz sobre los votantes menos comprometidos y con menor nivel de educación e interés político. Es decir, que los electores con una probabilidad a priori de votar más baja son los que mejor responden a la persuasión que los partidos buscan con la campaña. Para comprobar la influencia de las características personales de los votantes en su conducta electoral, la sección tercera propone un modelo que relaciona esas características con los diferentes efectos de las campañas.

Los trabajos académicos sobre las campañas para las elecciones generales en España son, hasta donde sé, los siguientes: Barreiro y Sánchez-Cuenca (1998); Lledó Callejón (2001) y Fernández Mellizo-Soto (2001), sobre las elecciones de 1993; Diez Nicolás y Semetko (1999) y Criado (2008), sobre las de 1996; Martínez i Coma (2008), sobre las campañas de 1993 y 2000; y Moreno Martínez (2010), sobre la campaña de 2008.

${ }^{1}$ Estudios 2.915 y 2.920 . El CIS entrevistó a unas 6.000 personas representativas de la población española de 18 o más años. Las entrevistas del estudio preelectoral tuvieron lugar entre el 6 y el 23 de octubre de 2011 y las del estudio postelectoral, entre el 18 de marzo y el 18 de abril de 2012. 
En este artículo aplico una estrategia empírica similar a la de Martínez i Coma (2008) para estimar los efectos de la campaña para las elecciones generales españolas de 2011 sobre los resultados de los tres partidos de ámbito nacional más votados: $\mathrm{PP}, \mathrm{PSOE}$ e IU $\mathrm{I}^{2}$. Los motivos para estudiar estas elecciones son tres. El primero es que las elecciones generales de 2011 se celebraron en un contexto especial, el de la más grave crisis económica que experimentó el país desde la restauración de la democracia. El segundo es el número de personas que decidió su conducta electoral durante la campaña. Según la encuesta postelectoral del CIS, fueron casi 8 millones frente a unos 6 o 6,5 millones que lo hicieron durante las campañas electorales de 2000 y 2008. El tercer motivo es que, hasta donde sé, los efectos de la campaña electoral de 2011 aun no han sido estimados.

El artículo está organizado como sigue: la sección segunda define los efectos de la campaña y estima su magnitud para las elecciones generales de 2011. La sección tercera discute los resultados de las estimaciones del modelo que relaciona los efectos de las campañas con las características individuales de los electores. La sección cuarta presenta las conclusiones. Los apéndices detallan la construcción de las variables del modelo y proporcionan su estadística descriptiva.

\section{Estimación de los efectos de la campaña}

Desde Lazarsfeld et al. (1944), la literatura distingue tres efectos de las campañas electorales sobre el comportamiento de los votantes:

Refuerzo: consiste en que la campaña reafirma la intención de voto inicial de los electores.

Activación: la campaña hace que terminen votando electores que estaban indecisos o pensaban abstenerse.

Conversión: la campaña cambia las preferencias de voto de algunos electores.

A estos tres efectos Martínez i Coma (2008) añade un cuarto:

Desactivación: algunos electores que pensaban votar, terminan absteniéndose por los sentimientos de alienación que les produce la campaña.

Para calcular estos cuatro efectos comparo la intención de voto de la encuesta

${ }^{2}$ Las elecciones generales se celebraron el 20 de noviembre de 2011. El presidente del Gobierno anunció el adelanto electoral a mediados de julio y disolvió las Cortes a finales de septiembre. La campaña electoral oficial transcurrió entre el 4 y el 18 de noviembre. Lo que se conoce como precampaña se extiende desde que se disuelven las Cortes hasta el comienzo de la campaña oficial. La movilización de los partidos se suele intensificar un mes antes del día de las votaciones. Las actividades de la precampaña y la campaña son funcionalmente equivalentes. 
preelectoral con el voto declarado en la encuesta postelectoral del panel del CIS para las elecciones generales de 2011. Los cambios que detecta este procedimiento pueden considerarse efectos plausibles de la campaña en tanto se producen durante un período de tiempo que incluye la campaña electoral. Esto no permite concluir, sin embargo, que los cambios observados se deban a la campaña ${ }^{3}$.

Pero, por otra parte, en la medida en que durante el período comprendido entre las dos encuestas el clima político general no registre alteraciones externas de importancia, parece razonable suponer que los efectos detectados al comparar la intención de voto con el voto declarado sean resultados del esfuerzo movilizador que realizan los partidos antes de unas elecciones. Informarse consume recursos y el rendimiento privado de la información política es muy bajo para la mayoría de la gente. En las campañas electorales, sin embargo, los partidos destacan sus realizaciones, dan a conocer sus propuestas y critican las de sus rivales. Asumen, de este modo, lo que Downs (1957: 222) denomina «los costes transferibles de votar», reduciendo las molestias que ocasiona reunir y analizar la información política. Por tanto, el hecho que la abundante información que circula durante las campañas abarate los costes de decisión del voto, permite interpretar los efectos observados en el comportamiento de los electores entre las dos encuestas electorales como efectos esperados de la campaña.

En otras palabras, los partidos emprenden las campañas para superar la apatía racional de los votantes. La publicidad, los actos en los distritos y los debates entre los candidatos, fomentan el interés y la participación en las urnas de los electores al ayudarles a elegir entre programas de gobierno alternativos (Jacobson, 1990; Shaw, 1999; Clinton y Lapinski, 2004). La abundancia de noticias que genera la campaña sobre los temas de la elección y los candidatos de los partidos puede cambiar las preferencias de voto iniciales. Pero puede también reforzarlas si los electores se informan en los medios afines a sus inclinaciones partidistas (Diez Nicolás y Semetko, 1999). Es decir, que la información es capaz de cambiar las actitudes previas de los electores, aunque también de reafirmarlas mediante la percepción y retención selectiva de los mensajes que se difunden (Canel, 1998).

La tabulación cruzada de la intención de voto declarada en la encuesta preelectoral del panel (pregunta 11) y del recuerdo de voto en la encuesta postelectoral (pregunta 36), figura en el Cuadro 1. El cuadro considera por separado al Partido Popular, el Partido Socialista e Izquierda Unida/Iniciativa por Cataluña (ICV) y agrupa el resto de los partidos en la categoría de «otros», lo que impide calcular los efectos de la campaña para estos partidos. En el panel del CIS solo hay suficientes observaciones para hacer una estimación fiable de los efectos de la campaña para los tres partidos más votados.

${ }^{3}$ Por ejemplo, los atentados terroristas del 11 de marzo de 2004 en los trenes de cercanías de Madrid durante los últimos días de la campaña electoral para las elecciones generales de 2004 afectaron notablemente al comportamiento de los votantes (MICHAVILA, 2005; BALI, 2007). Sería falaz, sin embargo, atribuir esos efectos a la campaña electoral. 


\section{CUADRO 1}

CORRESPONDENCIA ENTRE INTENCIÓN DE VOTO (FILAS) Y RECUERDO DE VOTO (COLUMNAS). ELECCIONES GENERALES DE 2011 (NÚMERO DE ENCUESTADOS)

\begin{tabular}{|l|r|r|r|r|r|r|}
\hline & \multicolumn{1}{|c|}{ IU } & \multicolumn{1}{c|}{ PP } & PSOE & Otros & Abstención & \multicolumn{1}{c|}{ Total } \\
\hline IU/ICV & 164 & 13 & 31 & 36 & 15 & 259 \\
\hline PP & 10 & 1.481 & 75 & 61 & 80 & 1.707 \\
\hline PSOE & 42 & 71 & 803 & 48 & 51 & 1.015 \\
\hline Otros & 23 & 53 & 28 & 373 & 31 & 508 \\
\hline Abstención & 19 & 54 & 41 & 31 & 291 & 436 \\
\hline No sabe todavía & 48 & 219 & 199 & 126 & 86 & 678 \\
\hline Total & 306 & 1.891 & 1.177 & 675 & 554 & $\mathrm{~N}=4.603$ \\
\hline
\end{tabular}

FUENTE: Elaboración propia a partir del panel del CIS para las elecciones generales de 2011 (estudios 2.915 y 2.920).

El porcentaje de indecisos en la muestra del Cuadro 1 es del 14,7 por 100 , (678/4.603), lo que representa unos 5,3 millones de electores del censo de 2011 (35,8 millones). Por otra parte, teniendo en cuenta que 1.130 encuestados eligieron a qué partido votar durante la campaña electoral oficial (pregunta 35 de la encuesta postelectoral) y que 200 optaron por abstenerse (pregunta 34a de la misma encuesta), el 21,7 por 100 de los 6.082 encuestados de la muestra del panel decidió su conducta electoral durante la campaña (1.330/6.082), unos 7,8 millones de electores. En resumen, las personas que decidieron su conducta electoral durante la campaña de 2011 pueden cifrarse entre 5,3 y 7,8 millones; unas cifras altas comparadas con las de las elecciones previas y un importante incentivo para la movilización electoral de los partidos.

El Cuadro 2 muestra el cálculo de los cuatro efectos de la campaña para las elecciones generales de 2011 .

\section{CUADRO 2}

EFECTOS DE LA CAMPAÑA ELECTORAL DE 2011 (NÚMERO DE ELECTORES EN LA MUESTRA Y PORCENTAJES)

\begin{tabular}{|l|l|c|}
\hline \multicolumn{1}{|c|}{ Efectos } & \multicolumn{1}{c|}{ Composición } & Total \\
\hline Refuerzo & $164+1.481+803+373+291$ & $3.112(67,6 \%)$ \\
\hline Activación & $19+54+41+31+48+219+199+126$ & $737(16 \%)$ \\
\hline Conversión & $13+31+36+10+75+61+42+71+48+23+53+28$ & $491(10,7 \%)$ \\
\hline Desactivación & $15+80+51+31+86$ & $263(5,7 \%)$ \\
\hline Total & & $4.603(100 \%)$ \\
\hline
\end{tabular}

FUENTE: Cuadro 1. 
El refuerzo de la intención inicial de voto afectó al 68 por 100 de los votantes y fue el efecto dominante de la campaña de 2011. Este resultado es similar al que encuentran los estudios realizados para distintos países y sistemas electorales (Finkel, 1993; Finkel y Schrott, 1995; Fernández Mellizo-Soto, 2001; Martínez i Coma, 2008: 159). Parece, por tanto, que la mayoría de los electores encuentra en la cobertura informativa de los medios de comunicación y en el marketing político de los partidos motivos que confirman su preferencia electoral previa. El análisis estadístico de los electores reforzados en 2011 muestra que pertenecían al hinterland ideológico de los tres partidos examinados y que tenían una buena opinión de sus respectivos candidatos a la presidencia del gobierno ${ }^{4}$.

El segundo efecto de la campaña por orden de importancia, la activación, afectó al 16 por 100 de los encuestados de la muestra. Durante la campaña, los partidos buscan movilizar a sus partidarios, aquellos electores indecisos cuyas propensiones latentes (aproximadas por la identificación ideológica y las simpatías políticas) los sitúan en la esfera de influencia del partido (Lazarsfeld et al., 1968; Finkel, 1993; Holbrook y McClurg, 2005). En la campaña de 2011, los activados por el PSOE e IU procedieron del entorno ideológico de ambos partidos, mientras que la mayor parte de los activados por el PP fueron electores centristas ${ }^{5}$.

El efecto de conversión de la campaña fue de menor magnitud que los de refuerzo y activación. Un 11 por 100 de los votantes se comportó de modo inconsecuente con la preferencia de voto declarada en la encuesta preelectoral ${ }^{6}$. En 2011 cambió el partido beneficiario de la conversión entre los electores de izquierda. El argumento de que la fragmentación del voto de izquierdas favorecía al PP transfirió votos de IU al PSOE en las tres elecciones generales previas (García Viñuela y Artés, 2012). Este argumento volvió a ser utilizado en la campaña socialista, aunque en esta ocasión resultó menos eficaz. IU también pidió el voto de los socialistas desencantados y el trasvase neto de votos entre los dos partidos se saldó a favor de IU, que recibió por esta vía un 3,6 por 100 de los votos a la coalición declarados en la encuesta postelectoral.

La desactivación afectó al 5 por 100 de los electores de la muestra. El porcentaje de los que manifestaron en la encuesta preelectoral que pensaban votar y decidieron finalmente no hacerlo fue similar para los tres partidos: 4,7 por $100(80 / 1.707)$ para el PP, 5 por $100(51 / 1.015)$ para el PSOE y 5,8 por $100(15 / 259)$ para IU. La edad media de los desactivados de los tres partidos fue más baja que la de los votantes. La tendencia de los electores de izquierda a la desmovilización (Barreiro, 2002; Molinas, 2007), apenas se aprecia en estas elecciones.

${ }^{4}$ La posición ideológica media de los reforzados en la encuesta preelectoral fue de 2,7 para IU, 3,5 para el PSOE y 6,7 para el PP. El rango de valores de la posición ideológica está comprendido entre 1 (extrema izquierda) y 10 (extrema derecha). La valoración media de los líderes políticos se situó entre 6,8 y 7,5 , en una escala de 0 (opinión muy mala) a 10 (opinión muy buena).

${ }^{5}$ Estos electores se situaban en la posición 5,2 de la escala ideológica.

${ }^{6}$ Esta cifra es similar a la que obtiene MARTÍNEZ I COMA (2008: 94) para las elecciones generales de 2000 y 1993, y unos 5 puntos porcentuales más alta que la que encuentra FERNÁNDEZ MELLIZO-SOTO (2001) para las elecciones generales de 1993. 
El impacto neto de la campaña sobre la participación electoral, medido por la diferencia entre los efectos de activación y desactivación, fue positivo y del orden del 10 por 100. La campaña resultó, por tanto, útil para movilizar a los votantes. El impacto neto fue positivo para los tres partidos estudiados y de mayor magnitud para IU (16,1 por 100) y el PSOE (15,4 por 100) que para el PP (9,7 por 100).

El Cuadro 3 muestra los efectos de la campaña de 2011 por partidos. El efecto principal de la campaña, el refuerzo de las preferencias políticas previas, fue más importante para el PP que para los otros partidos del cuadro. La mayor estabilidad comparativa del electorado del PP puede deberse a la falta de sustitutivos cercanos electoralmente viables en el espacio ideológico de la derecha. Por otra parte, el contexto en el que se celebraron las elecciones de 2011 contribuyó a que el PP obtuviera una mayoría absoluta en las dos cámaras de las Cortes Generales. Cuando se convocaron las elecciones la situación del PSOE, el partido gobernante, era muy desfavorable. Un 90 por 100 de los encuestados por el CIS calificaban la situación económica de mala o muy mala (pregunta 4 de la encuesta preelectoral) y un 62 por 100 desaprobaba la gestión del gobierno (pregunta 5 de la misma encuesta). Estas circunstancias permitieron al PP ganar apoyos entre los electores indecisos del centro político, la zona en la que compite por los votos con el PSOE.

\section{CUADRO 3}

EFECTOS DE LAS CAMPAÑAS POR PARTIDOS (EN PORCENTAJE DEL VOTO DECLARADO AL PARTIDO). ELECCIONES DE 2011

\begin{tabular}{|l|c|c|c|c|}
\hline \multicolumn{1}{|c|}{ Partido } & Refuerzo & Activación & Conversión & Total \\
\hline PP & $1.481 / 1.891$ & $(219+54) / 1,891$ & $(13+71+53) / 1.891$ & 1.891 \\
& $(78,3 \%)$ & $(14,4 \%)$ & $(7,3 \%)$ & $(100)$ \\
\hline PSOE & $803 / 1.177$ & $(199+41) / 1177$ & $(31+75+28) / 1.177$ & 1.177 \\
& $(68,2 \%)$ & $(20,4 \%)$ & $(11,4 \%)$ & $(100)$ \\
\hline IU/ICV & $164 / 306$ & $(48+19) / 306$ & $(10+42+23) / 306$ & 306 \\
& $(53,6 \%)$ & $(21,9 \%)$ & $(24,5 \%)$ & $(100)$ \\
\hline
\end{tabular}

FUENTE: Cuadro 1.

Como muestra el Cuadro 3, los cambios en las preferencias políticas durante la campaña, los efectos de activación y conversión, fueron más intensos para los dos partidos de izquierda. La conversión aportó a IU la cuarta parte de los votos, que procedieron en su mayor parte del electorado socialista. La pérdida de confianza en el partido gobernante durante los dos últimos años de la legislatura animó a la nueva dirección de IU a organizar una campaña destinada a recuperar el peso electoral y parlamentario que había perdido desde 1996. Y la activación de sus propios simpatizantes le aportó una ganancia de votos de magnitud algo menor.

Las características de los votantes activados por la campaña de IU y las de los que pensando votar al inicio de la campaña al PSOE terminaron votando a IU, fueron 
muy similares por lo que se refiere a la edad (unos 45 años) y nivel de estudios (casi secundarios). La posición ideológica de los activados $(3,0)$ fue más próxima a la de IU $(2,4)$ que la de los convertidos $(3,5)$, más cercana a la del PSOE $(4,1)$.

El PSOE consiguió frenar con la campaña el duro revés que sufrió en estas elecciones, en las que perdió 4,5 millones de votantes y 59 escaños en el Congreso de los Diputados. Se puede estimar que la movilización para activar el voto ideológico de sus simpatizantes aportó al partido la quinta parte de los votos socialistas declarados en la encuesta postelectoral. Es decir, que el partido logró activar el apoyo de 1,4 millones de votantes que antes de iniciarse la campaña no sabían si acudirían a las urnas o manifestaron la intención de abstenerse. El análisis estadístico de estos votantes revela que tenían una edad media de 50 años, un nivel de estudios entre primarios y secundarios, y se ubicaban en la posición 4 de la escala ideológica, la misma en la que el conjunto de los encuestados por el CIS sitúa al Partido Socialista.

\section{El modelo: expectativas y resultados}

Los Cuadros 2 y 3 muestran la fuerza relativa de los cuatro efectos estimados de la campaña, pero nada permiten concluir sobre los factores de los que dependen. Para examinar el papel de los factores individuales propongo un modelo que relaciona la probabilidad relativa de los distintos efectos con algunas características sociológicas de los electores y con los cambios operados en sus actitudes durante el período electoral.

$$
Y=a+b X+c \Delta Z+\varepsilon
$$

La variable dependiente $(Y)$ está formada por cuatro categorías, que corresponden a los efectos de refuerzo, activación, conversión y desmovilización de la campaña. Se construye con los datos individuales de los electores que figuran agrupados en las celdas centrales del Cuadro 2. La estructura de la variable es la que muestra la última columna del mismo cuadro.

Las variables explicativas incluyen algunas características personales de los electores $(X)$ y los cambios en sus actitudes y valoraciones políticas $(Z)$ durante la campaña.

El impacto de las campañas no es igual para todos los votantes. Shaw (1999) muestra que las campañas influyen en mayor medida sobre los votantes con identificación partidista débil, y Kenny y McBurnett (1994) y Criado (2008) encuentran que la persuasión publicitaria es más eficaz sobre los electores con menor nivel de estudios e interés por las elecciones, porque son más fáciles de convencer que quienes tienen preferencias políticas definidas.

Las variables que forman el vector $X$ comprenden la edad, los estudios y el nivel de información de los encuestados. La edad, la educación formal y las decisiones mejor informadas propician preferencias menos permeables a los efectos de la per- 
suasión publicitaria de los partidos, al menos en el corto período de tiempo que separa la campaña electoral del día de las votaciones. Esperamos, por tanto, que las tres variables tengan un coeficiente negativo ${ }^{7}$ en los modelos que comparan los efectos de cambio de la campaña con la estabilidad de las preferencias políticas que representa el efecto de refuerzo.

Los cambios en las actitudes y valoraciones individuales durante la campaña $(\Delta Z)$ incluyen las variaciones en la ideología, en la percepción de la situación económica y en la valoración de los candidatos de los tres partidos más votados a la presidencia del gobierno: Mariano Rajoy, Alfredo Pérez Rubalcaba y Cayo Lara. La importancia de la ideología en la emisión del voto ha sido destacada por los autores que han investigado el comportamiento de los votantes españoles (Torcal y Chhibber, 1997; Lago, 2005). Los estudios electorales también encuentran que sobre el voto influyen tanto la situación de la economía nacional (Fiorina, 1981; Fraile, 2005; Fraile y Beck, 2010), como la imagen que tienen los votantes de los candidatos de los partidos (Bean y Mughan, 1989; Stewart y Clarke, 1992; Rico, 2009). Además, la información sobre los candidatos que se difunde durante la campaña electoral es probable que modifique las valoraciones de los electores (Gelman y King, 1993).

Esperamos, por tanto, que la divergencia entre la intención de voto expresada en la encuesta preelectoral y el voto declarado en la encuesta postelectoral sea más acusada en los individuos cuyas actitudes y percepciones cambian durante la movilización que emprenden los partidos para captar el voto. En otras palabras, las variaciones en la ideología y en la opinión de los votantes sobre la situación económica y los candidatos de los partidos, deberían propiciar los efectos de cambio de la campaña frente al refuerzo, por lo que esperamos coeficientes positivos para estas variables.

Los datos de las características personales de los votantes y su variación durante el período que comprende la campaña electoral, proceden del panel del CIS sobre las elecciones generales de 2011. El Apéndice 1 describe la construcción de las variables individuales y el Apéndice 2 proporciona su estadística descriptiva.

Para facilitar la interpretación de los coeficientes de las variables del modelo, utilizo cuatro probits binomiales. Las cuatro variables dependientes dicotómicas son las siguientes: $1 .^{\mathrm{a}}$, resto de efectos (1) versus refuerzo $(0) ; 2 .{ }^{\mathrm{a}}$, activación (1) versus refuerzo (0); 3. ${ }^{\mathrm{a}}$, conversión (1) versus refuerzo (0); y 4. ${ }^{\mathrm{a}}$, desactivación (1) versus refuerzo $(0)$.

\footnotetext{
${ }^{7}$ Como la información puede modificar las preferencias de voto iniciales, el coeficiente de la variable en los modelos de regresión que usan el refuerzo como categoría de referencia podría ser positivo. Cabe esperar que la información que circula durante la campaña electoral reafirme las preferencias previas de voto en unos casos y las cambie en otros. Como no hay razones para anticipar el efecto que predomina, el signo del coeficiente es una cuestión empírica.
} 


\section{CUADRO 4}

\section{COEFICIENTES PROBIT Y EFECTOS MARGINALES DE LAS VARIABLES SIGNIFICATIVAS. ELECCIONES GENERALES DE 2011}

\begin{tabular}{|c|c|c|c|c|}
\hline Variables & $\begin{array}{l}\text { Resto de } \\
\text { efectos } \\
\text { versus } \\
\text { refuerzo }\end{array}$ & $\begin{array}{l}\text { Activación } \\
\text { versus } \\
\text { refuerzo }\end{array}$ & $\begin{array}{l}\text { Conversión } \\
\text { versus } \\
\text { refuerzo }\end{array}$ & $\begin{array}{c}\text { Desactivación } \\
\text { versus } \\
\text { refuerzo }\end{array}$ \\
\hline Edad & $\begin{array}{c}-0,0070 * * * \\
(0,0026) \\
-0,0023\end{array}$ & $\begin{array}{l}-0,0003 \\
(0,0031)\end{array}$ & $\begin{array}{c}-0,0087 * * * \\
(0,0034) \\
-0,0018\end{array}$ & $\begin{array}{c}-0,0256 * * * \\
(0,0063) \\
-0,0013\end{array}$ \\
\hline Estudios & $\begin{array}{c}0,0095 \\
(0,0439)\end{array}$ & $\begin{array}{c}0,0223 \\
(0,0535)\end{array}$ & $\begin{array}{c}0,0026 \\
(0,0564)\end{array}$ & $\begin{array}{l}-0,0235 \\
(0,0965)\end{array}$ \\
\hline Información & $\begin{array}{c}-0,2557 * * * \\
(0,0793) \\
-0,0819 \\
\end{array}$ & $\begin{array}{c}-0,3170 * * * \\
(0,1006) \\
-0,0697 \\
\end{array}$ & $\begin{array}{l}-0,0703 \\
(0,1031)\end{array}$ & $\begin{array}{c}-0,4867 * * * \\
(0,1576) \\
-0,0246 \\
\end{array}$ \\
\hline Cambio ideológico & $\begin{array}{c}0,0801 * * * \\
(0,0343) \\
0,0257\end{array}$ & $\begin{array}{c}0,0396 \\
(0,0424)\end{array}$ & $\begin{array}{c}0,1131 * * * \\
(0,0430) \\
0,0229\end{array}$ & $\begin{array}{c}0,0631 \\
(0,0771)\end{array}$ \\
\hline $\begin{array}{l}\text { Cambio en la percepción de la } \\
\text { economía }\end{array}$ & $\begin{array}{c}0,0003 \\
(0,0597)\end{array}$ & $\begin{array}{c}0,0078 \\
(0,0731)\end{array}$ & $\begin{array}{l}-0,0497 \\
(0,0777)\end{array}$ & $\begin{array}{c}0,1652 \\
(0,1229)\end{array}$ \\
\hline $\begin{array}{l}\text { Cambio en la valoración de Mariano } \\
\text { Rajoy }\end{array}$ & $\begin{array}{c}0,0805^{* * *} \\
(0,0257) \\
0,0258\end{array}$ & $\begin{array}{c}0,0591 * \\
(0,0317) \\
0,0130\end{array}$ & $\begin{array}{c}0,0809 * * * \\
(0,0326) \\
0,0164\end{array}$ & $\begin{array}{c}0,0901 * \\
(0,0539) \\
0,0046\end{array}$ \\
\hline $\begin{array}{l}\text { Cambio en la valoración de Alfredo } \\
\text { Pérez Rubalcaba }\end{array}$ & $\begin{array}{c}0,0854 * * * \\
(0.0251) \\
0.0274\end{array}$ & $\begin{array}{c}0,0591 * * \\
(0,0311) \\
0,0130\end{array}$ & $\begin{array}{c}0,0104 * * * \\
(0,0316) \\
0,0211\end{array}$ & $\begin{array}{c}0,0537 \\
(0,0523)\end{array}$ \\
\hline $\begin{array}{l}\text { Cambio en la valoración de Cayo } \\
\text { Lara }\end{array}$ & $\begin{array}{l}-0,0042 \\
(0,0258)\end{array}$ & $\begin{array}{l}-0,0250 \\
(0,0325)\end{array}$ & $\begin{array}{c}0,0238 \\
(0,0318)\end{array}$ & $\begin{array}{c}0,0043 \\
(0,0563)\end{array}$ \\
\hline Constante & $\begin{array}{c}-0,4051 * * \\
(0,1814)\end{array}$ & $\begin{array}{c}-0,9772 * * * \\
(0,2277)\end{array}$ & $\begin{array}{l}-1,0490 * * * \\
(0,2315)\end{array}$ & $\begin{array}{l}-0,6417 * \\
(0,3750)\end{array}$ \\
\hline $\mathrm{N}$ & 1.461 & 1.259 & 1.244 & 1.118 \\
\hline LR chi2 (8) & 59,86 & 21,51 & 44,12 & 40,73 \\
\hline Prob > chi 2 & 0,0000 & 0,0059 & 0,0000 & 0,0000 \\
\hline Pseudo R2 & 0,0357 & 0,0209 & 0,0455 & 0,1228 \\
\hline$\%$ casos predichos & 74,5 & 85,8 & 86,9 & 96,6 \\
\hline
\end{tabular}

NOTA: La probabilidad del refuerzo de la intención de voto es la categoría de referencia en los cuatro casos. Los errores estándar figuran entre paréntesis; los efectos marginales están en cursiva. Niveles de significación: * p $<0,10$; $* * \mathrm{p}<0,05 ; * * * \mathrm{p}<0,01$. 
El Cuadro 4 muestra los coeficientes de las cuatro estimaciones del modelo. La referencia en todos los casos es el refuerzo de la intención de voto. Por tanto, los coeficientes hay que interpretarlos en términos relativos, como un aumento (si son positivos) o una disminución (si son negativos) de la probabilidad de que se produzca alguno de los efectos de cambio de la campaña comparado con la ausencia de cambio que representa el refuerzo. Los datos que figuran en cursiva debajo de los errores estándar de los coeficientes significativos son los efectos marginales para un individuo medio de la muestra. Es decir, que miden el impacto marginal de cada variable significativa del modelo cuando las demás variables independientes se mantienen en sus valores medios.

Los electores con más años, información y nivel de estudios deberían ser más resistentes a la propaganda política, por lo que esperíamos que estos factores favorecieran la estabilidad de las preferencias (el refuerzo) frente a los efectos de cambio de la campaña (los demás). Las estimaciones del Cuadro 4 confirman estas expectativas para la edad y el nivel de información de los electores. Ambas variables tienen siempre el signo negativo esperado y son significativas en tres de las cuatro estimaciones. No hay pruebas, sin embargo, de que el nivel de estudios sea un factor explicativo de los efectos de cambio de la campaña. Choi (2009) mantiene que la información de los votantes es más relevante que su grado de educación formal, y que la influencia de la variable estudios desaparece cuando se controla por el nivel de información. Las estimaciones del Cuadro 4 concuerdan con la opinión de Choi. Los coeficientes de la variable estudios no tienen el signo anticipado y están lejos ser significativos.

El cambio en la ideología de los encuestados opera en todas las estimaciones en la dirección esperada; es decir, favoreciendo los otros efectos de la campaña frente al mantenimiento de la opción inicial de voto. El coeficiente del cambio ideológico es significativo para la conversión, aunque no para los efectos de activación y desmovilización. Las estimaciones no muestran, sin embargo, que los cambios durante la campaña en la percepción de la situación económica del país afectaran al comportamiento electoral.

Las variaciones en la opinión sobre los candidatos de los partidos durante la campaña deberían favorecer el cambio de la intención de voto expresada antes de iniciarse la campaña. Los resultados de la estimación del modelo muestran que esto ha sido así para los candidatos a la presidencia del gobierno de los dos partidos mayoritarios. En ambos casos, las diferencias en la actitud hacia los líderes del PP y del PSOE durante el período que se extiende entre las dos encuestas del panel electoral, tuvieron un impacto positivo y significativo sobre la activación y la conversión de los electores respecto al refuerzo.

En resumen, encuentro que la edad, el nivel de información de los electores y los cambios en la valoración de los candidatos de los dos partidos mayoritarios, contribuyen a explicar la fuerza relativa de los diferentes efectos de la campaña. Las estimaciones muestran que la influencia de estos factores no se produce de forma aleatoria, aunque sus efectos marginales son pequeños. 


\section{Conclusiones}

El análisis estadístico revela que la campaña para las elecciones generales de 2011 promovió la participación electoral. El efecto dominante de la campaña, el refuerzo de las preferencias de voto previas, operó básicamente en el hinterland ideológico de los tres partidos más votados (PP, PSOE e IU). El efecto de refuerzo fue de mayor magnitud para el PP, el principal partido de la oposición y ganador de las elecciones. La pérdida de confianza en el PSOE, el partido gobernante en la legislatura anterior, permitió al PP activar el apoyo de los electores indecisos del centro del espacio político, en la zona en la que compite por los votos con el Partido Socialista.

Si medimos la importancia de la campaña por los cambios en las preferencias expresadas en la encuesta preelectoral, esos cambios fueron mayores para los dos partidos de izquierda. El efecto de conversión entre los votantes de izquierda, que en la década pasada benefició al PSOE mediante el mecanismo del voto estratégico, favoreció en esta ocasión a IU, que recuperó algo más de la mitad de la fuerza electoral y parlamentaria que alcanzó en los años noventa. La movilización del voto ideológico de sus partidarios permitió al PSOE contener la notable caída de apoyo popular que tuvo en estas elecciones.

La estimación del modelo que relaciona los efectos de la campaña con las características personales de los votantes, encuentra que la edad y el nivel de información política promovieron el refuerzo de la intención de voto anterior a la campaña. Por el contrario, el cambio ideológico y las diferencias en la valoración de los candidatos a la presidencia del gobierno del PP y del PSOE entre las dos encuestas del panel electoral, favorecieron los efectos de cambio de la campaña. La influencia de las variables individuales en la explicación de estos efectos, aunque significativa, fue débil.

El procedimiento que utiliza este estudio atribuye la volatilidad detectada en las preferencias electorales entre las dos encuestas del panel a la campaña. Es probable, sin embargo, que esa volatilidad refleje en parte las dudas e incertidumbres de los electores derivadas del agudo desgaste del partido gobernante y las sombrías perspectivas económicas en que se celebraron las elecciones generales de 2011. La cautela aconseja, por tanto, no extrapolar los resultados que encuentra este trabajo a otras campañas electorales.

Por último, los efectos estimados no tienen en cuenta que la intensidad de la campaña depende de las presiones competitivas que soportan los partidos en los distritos. La investigación posterior puede aclarar en qué medida la movilización diferencial en los distritos influye sobre los diferentes efectos de la campaña. También sería interesante comprobar el impacto en la producción de estos efectos de las instituciones electorales que condicionan la viabilidad de los partidos en los distritos. 


\section{Referencias bibliográficas}

[1] BALI, VALENTINA (2007): «Terror and elections: Lessons from Spain», Electoral Studies, 26: 669-687.

[2] BARREIRO, BELÉN (2002): «La progresiva desmovilización de la izquierda en España: un análisis de la abstención en las elecciones generales de 1986 a 2000», Revista Española de Ciencia Política, 6: 183-205.

[3] BARREIRO, BELÉN y SÁNCHEZ-CUENCA, IGNACIO (1998): «Análisis del cambio de voto hacia el PSOE en las elecciones de 1993», Revista Española de Investigaciones Sociológicas, 82: 191-211.

[4] BEAN, CLIVE y MUGHAN, ANTHONY (1989): «Leadership effects in parliamentary elections in Australia and Britain», American Political Science Review, 83: 1165-1180.

[5] CAMPBELL, ANGUS; CONVERSE, PHILIP; MILLER, WARREN, y STOKES, DONALD (1960): The American voter. Chicago: The University of Chicago Press.

[6] CANEL, MARÍA JOSÉ (1998): «Los efectos de las campañas electorales”, Comunicación y Sociedad, 1: 47-67.

[7] CHOI, JUNGUG (2009): «Strategic voting in India: Its extent and determinants in the 2004 general election», Asian Survey, 49: 609-624.

[8] CLINTON, JOSHUA y LAPINSKI, JOHN (2004): “'Targeted' advertising and voter turnout: An experimental study of the 2000 Presidential election», Journal of Politics, 66: 69-96.

[9] CRIADO, HENAR (2008): «The effects of party mobilization strategies on the vote: The PSOE and the PP in the 1996 Spanish election», European Journal of Political Research, 47: 80-100.

[10] DÍEZ NICOLÁS, JUAN y SEMETKO, HOLLI (1995): «Los programas de noticias de televisión y las campañas electorales de 1993 y 1996», en Muñoz Alonso, Alejandro y Rospir, Ignacio (eds.), Democracia mediática y campañas electorales. Barcelona: Ariel, 151-201.

[11] DOWNS, ANTHONY (1957): An economic theory of democracy. New York: Harper \& Row.

[12] FERNÁNDEZ MELLIZO-SOTO, MARÍA (2001): «¿Para qué sirven las campañas electorales?: Los efectos de la campaña electoral española de 1993», Revista Española de Investigaciones Sociológicas, 93: 61-87.

[13] FINKEL, STEVEN (1993): «Reexamining the 'minimal effects' model in Presidential electoral campaigns», Journal of Politics, 55: 1-21.

[14] FINKEL, STEVEN y SCHROTT, PETER (1995): «Campaign effects on voter choice in the German election of 1990», British Journal of Political Science, 23: 349-377.

[15] FIORINA, MORRIS (1981): Retrospective voting in American national elections. New Haven: Yale University Press.

[16] FRAILE MALDONADO, MARTA (2005): Cuando la economía entra en las urnas. Madrid: Centro de Investigaciones Sociológicas.

[17] FRAILE, MARTA y LEWIS-BECK, MICHAEL (2010): «Economic voting in Spain: A 2000 panel test», Electoral Studies, 29: 210-220.

[18] FRANZ, MICHAEL y RIDEOUT, TRAVIS (2007): «Does political advertising persuade?", Political Behavior, 29: 465-491. 
[19] GARCÍA VIÑUELA, ENRIQUE y ARTÉS, JOAQUÍN (2012): «Strategic voting and effective representation thresholds: Evidence from three Spanish general elections», European Journal of Political Research, 51: 289-315.

[20] GELMAN, ANDREW y KING, GARY (1993): «Why are American presidential election campaign polls so variable when voters are so predictable», British Journal of Political Science, 23: 409-451.

[21] HILLYGUS, SUNSHINE (2005): «Campaign effects and the dynamics of turnout intention in election 2000», Journal of Politics, 67: 50-68.

[22] HOLBROOK, THOMAS (1996): Do campaigns matter? Thousand Oaks: Sage Publications.

[23] HOLBROOK, THOMAS y MCCLURG, SCOTT (2005): «The mobilization of core supporters: Campaigns, turnout and electoral competition in US presidential elections», American Journal of Political Science, 49: 689-703.

[24] JACOBSON, GARY (1990). «The effects of campaign spending in House elections: new evidence for old arguments», American Political Science Review, 34: 334-362.

[25] KENNY, CHRISTOPHER y McBURNETT, MICHAEL (1994): «An individual level multiequation model of expenditure effects in contested House elections», American Political Science Review, 88: 699-707.

[26] LAGO PEÑAS, IGNACIO (2005): El voto estratégico en las elecciones generales en España, 1977-2000. Madrid: Centro de Investigaciones Sociológicas.

[27] LAZARSFELD, PAUL; BERELSON, BERNARD y GAUDET, HAZEL (1968): The people's choice: How the voter makes up his mind in a presidential election, 3. ${ }^{\mathrm{a}}$ ed. (1. ${ }^{a}$ ed. 1944). New York: Columbia University Press.

[28] LLEDÓ CALLEJÓN, PABLO (2001): «La influencia de los debates electorales sobre la decisión de voto: el caso de mayo de 1993 en España», Revista Española de Ciencia Política, 5: 143-170.

[29] McCLURG, SCOTT y HOLBROOK, THOMAS (2009): «Living in a battleground: Presidential campaigns and fundamental predictors of vote choice», Political Research Quarterly, 62: 495-506.

[30] MARTÍNEZ I COMA, FERRÁN (2008): Por qué importan las campañas electorales. Madrid: Centro de Investigaciones Sociológicas.

[31] MICHAVILA, NARCISO (2005): «War, terrorism and elections: Electoral impacts of the Islamic terror attacks on Madrid», Working Paper, 13. Madrid: Real Instituto Elcano.

[32] MOLINAS, CÉSAR (2007): «El poder decisorio de la izquierda volátil», El País, $11 / 11 / 2007$.

[33] MORENO MARTÍNEZ, CRISTINA (2010): «El efecto de la campaña para las elecciones generales españolas de 2008 sobre la información política y la participación electoral de los votantes", Revista Española de Ciencia Política, 24: 53-81.

[34] POPKIN, SAMUEL (1991): The reasoning voter: Communication and persuasion in presidential campaigns. Chicago: University of Chicago Press.

[35] RICO, GUILLEM (2009): Líderes políticos, opinión pública y comportamiento electoral en España. Madrid: Centro de Investigaciones Sociológicas.

[36] ROSENTONE, STEVEN y HANSEN, JOHN (1993): Mobilization, participation and democracy in America. New York: Macmillan.

[37] SIMON, ADAM (2002): The winning message: candidate behavior, campaign discourse and democracy. New York: Cambridge University Press. 
[38] SHAW, DARON (1999): «The methods behind the madness: Presidential Electoral College strategies», Journal of Politics, 61: 893-913.

[39] STEWART, MARIANNE y CLARKE, HAROLD (1992): «The (un)importance of party leaders: Leader images and party choice in the 1987 British election», Journal of Politics, 54: 447-470.

[40] TORCAL, MARIANO y CHHIBBER, PRADEEP (1997): «Elite strategy, social cleavages and party systems in a new democracy: Spain», Comparative Political Studies, 30: $27-54$. 


\section{Apéndice 1 \\ DESCRIPCIÓN DE LAS VARIABLES}

- Cambio en la percepción de la situación económica: diferencia en valor absoluto entre la percepción del encuestado sobre la economía del país antes y después de la campaña (pregunta 4 de la encuesta preelectoral y pregunta 8 de la postelectoral). El recorrido de ambas preguntas va de 1 (percepción muy mala) a 5 (percepción muy buena).

- Cambio ideológico: diferencia en valor absoluto entre la posición del encuestado en la escala izquierda-derecha antes de la campaña (pregunta 25 de la encuesta preelectoral) y después de la campaña (pregunta 40 de la encuesta postelectoral). El recorrido de la escala ideológica va de 1 (extrema izquierda) a 10 (extrema derecha).

- Cambio en la valoración de los candidatos a la presidencia del gobierno del Partido Popular, Partido Socialista e Izquierda Unida, antes de la campaña y después de las elecciones: diferencia en valor absoluto en la valoración de Mariano Rajoy (pregunta 1708 de la encuesta preelectoral y 4213 de la postelectoral), Alfredo Pérez Rubalcaba (preguntas 1707 y 4212) y Cayo Lara (preguntas 1706 y 4210). La codificación de ambas preguntas va de 1 (valoración muy mala del candidato) a 10 (valoración muy buena).

- Edad: años del encuestado (pregunta 31 de la encuesta preelectoral del panel).

- Efectos de la campaña electoral: variable dependiente general. Se construye a partir de la información que contiene el Cuadro 1 del texto. Variable discreta con cuatro categorías, que corresponden a los cuatro efectos de la campaña: refuerzo (1), activación (2), conversión (3) y desactivación (4). Las cuatro variables dependientes de los modelos estimados son: 1 a $^{\mathrm{a}}$, resto de efectos (1) versus refuerzo (0); 2. ${ }^{\mathrm{a}}$, activación (1) versus refuerzo (0); 3 . $^{\mathrm{a}}$, conversión (1) versus refuerzo (0); y 4..$^{\mathrm{a}}$, desactivación (1) versus refuerzo (0).

- Estudios: nivel de estudios del encuestado. Sin estudios (0), estudios primarios (1), estudios secundarios o formación profesional (2), estudios universitarios (3) (pregunta 61a de la encuesta postelectoral del panel).

- Información: nivel de información política del encuestado. Bajo (0), medio (1), alto (2). Se construye sumando las preguntas 2501, 2502 y 2503 de la encuesta postelectoral del panel. 


\section{Apéndice 2}

ESTADÍSTICA DESCRIPTIVA DE LAS VARIABLES INDEPENDIENTES

\begin{tabular}{|l|c|c|c|c|c|}
\hline Variable & $\mathrm{N}$ & Media & \multicolumn{1}{|c|}{$\sigma$} & Min. & Max. \\
\hline Edad & 6.082 & 47,766 & 17,655 & 18 & 95 \\
\hline Estudios & 6.072 & 1,682 & 0,839 & 0 & 3 \\
\hline Información & 6.082 & 0,717 & 0,524 & 0 & 2 \\
\hline Cambio ideológico & 4.756 & 0,982 & 1,159 & 0 & 9 \\
\hline Cambio en la percepción de la economía & 6.010 & 0,515 & 0,623 & 0 & 4 \\
\hline $\begin{array}{l}\text { Cambio en la valoración de Mariano } \\
\text { Rajoy }\end{array}$ & 5.471 & 1,620 & 1,685 & 0 & 10 \\
\hline $\begin{array}{l}\text { Cambio en la valoración de Alfredo } \\
\text { Pérez Rubalcaba }\end{array}$ & 5.284 & 1,604 & 1,634 & 0 & 10 \\
\hline Cambio en la valoración de Cayo Lara & 1.942 & 1,469 & 1,477 & 0 & 10 \\
\hline
\end{tabular}

